\title{
DECOMPOSITION OF COMPLETE BIPARTITE MULTIGRAPHS INTO PATHS AND CYCLES HAVING $k$ EDGES
}

\author{
Shanmugasundaram JEEvadoss \\ AND \\ Appu Muthusamy \\ Periyar University \\ Salem, Tamil Nadu \\ INDIA \\ e-mail: raazdoss@gmail.com \\ ambdu@yahoo.com
}

\begin{abstract}
We give necessary and sufficient conditions for the decomposition of complete bipartite multigraph $K_{m, n}(\lambda)$ into paths and cycles having $k$ edges. In particular, we show that such decomposition exists in $K_{m, n}(\lambda)$, when $\lambda \equiv 0$ $(\bmod 2), m, n \geq \frac{k}{2}, m+n>k$, and $k(p+q)=2 m n$ for $k \equiv 0(\bmod 2)$ and also when $\lambda \geq 3, \lambda m \equiv \lambda n \equiv 0(\bmod 2), k(p+q)=\lambda m n, m, n \geq k$, (resp., $m, n \geq 3 k / 2)$ for $k \equiv 0(\bmod 4)($ respectively, for $k \equiv 2(\bmod 4))$. In fact, the necessary conditions given above are also sufficient when $\lambda=2$.
\end{abstract}

Keywords: path, cycle, graph decomposition, multigraph.

2010 Mathematics Subject Classification: 05C38, 05C51.

\section{REFERENCES}

[1] A.A. Abueida and M. Daven, Multidesigns for graph-pairs of order 4 and 5, Graphs Combin. 19 (2003) 433-447. doi:10.1007/s00373-003-0530-3

[2] A.A. Abueida and M. Daven, Multidecompositions of the complete graph, Ars Combin. 72 (2004) 17-22.

[3] A.A. Abueida, M. Daven and K.J. Roblee, Multidesigns of the $\lambda$-fold complete graphpairs of orders 4 and 5, Australas. J. Combin. 32 (2005) 125-136. 
[4] A.A. Abueida and T. O'Neil, Multidecomposition of $K_{m}(\lambda)$ into small cycles and claws, Bull. Inst. Comb. Appl. 49 (2007) 32-40.

[5] A.A. Abueida and C. Hampson, Multidecomposition of $K_{n}-F$ into graph-pairs of order 5 where $F$ is a Hamilton cycle or an (almost) 1-factor, Ars Combin. 97 (2010) 399-416.

[6] A.A. Abueida and M. Daven, Multidecompositions of several graph products, Graphs Combin. 29 (2013) 315-326.

doi:10.1007/s00373-011-1127-x

[7] A.A. Abueida and C. Lian, On the decompositions of complete graphs into cycles and stars on the same number of edges, Discuss. Math. Graph Theory 34 (2014) 113-125. doi:10.7151/dmgt.1719

[8] J.A. Bondy and U.R.S. Murty, Graph Theory with Applications (The Macmillan Press Ltd, New York, 1976).

[9] C.C. Chou, C.M. Fu and W.C. Huang, Decomposition of $K_{m, n}$ into short cycles, Discrete Math. 197/198 (1999) 195-203. doi:10.1016/S0012-365X(99)90063-8

[10] C.C. Chou and C.M. Fu, Decomposition of $K_{m, n}$ into 4-cycles and 2 -cycles, J. Comb. Optim. 14 (2007) 205-218. doi:10.1007/s10878-007-9060-x

[11] S. Jeevadoss and A. Muthusamy, Sufficient condition for $\left\{C_{4}, C_{2 t}\right\}$-decomposition of $K_{2 m, 2 n}-A n$ improved bound, S. Arumugam and B. Smyth (Eds.), Combinatorial Algorithms, IWOCA 2012, LNCS, (Springer-Verlag Berlin Heidelberg) 7643 (2012) $143-147$.

[12] S. Jeevadoss and A. Muthusamy, Decomposition of complete bipartite graphs into paths and cycles, Discrete Math. 331 (2014) 98-108. doi:10.1016/j.disc.2014.05.009

[13] H.-C. Lee and Y.-P. Chu Multidecompositions of the balanced complete bipartite graphs into paths and stars, ISRN Combinatorics (2013). doi:10.1155/2013/398473

[14] H.-C. Lee, Multidecompositions of complete bipartite graphs into cycles and stars, Ars Combin. 108 (2013) 355-364.

[15] H.-C. Lee, Decomposition of complete bipartite graphs with a 1-factor removed into cycles and stars, Discrete Math. 313 (2013) 2354-2358. doi:10.1016/j.disc.2013.06.014

[16] D.G. Sarvate and L. Zhang, Decomposition of a $\lambda K_{v}$ into equal number of $K_{3} s$ and $P_{3} s$, Bull. Inst. Comb. Appl. 67 (2013) 43-48.

[17] T.-W. Shyu, Decompositions of complete graphs into paths and cycles, Ars Combin. 97 (2010) 257-270. 
[18] T.-W. Shyu, Decompositions of complete graphs into paths and stars, Discrete Math. 330 (2010) 2164-2169.

doi:10.1016/j.disc.2010.04.009

[19] T.-W. Shyu, Decompositions of complete graphs into paths of length three and triangles, Ars Combin. 107 (2012) 209-224.

[20] T.-W. Shyu, Decomposition of complete graphs into cycles and stars, Graphs Combin. 29 (2013) 301-313. doi:10.1007/s00373-011-1105-3

[21] T.-W. Shyu, Decomposition of complete bipartite graphs into paths and stars with same number of edges, Discrete Math. 313 (2013) 865-871. doi:10.1016/j.disc.2012.12.020

[22] D. Sotteau, Decomposition of $K_{m, n}\left(K_{m, n}^{*}\right)$ into cycles (circuits) of length $2 k$, J. Combin. Theory Ser. B 30 (1981) 75-81. doi:10.1016/0095-8956(81)90093-9

[23] M. Truszczyński, Note on the decomposition of $\lambda K_{m, n}\left(\lambda K_{m, n}^{*}\right)$ into paths, Discrete Math. 55 (1985) 89-96.

doi:10.1016/S0012-365X(85)80023-6

Received 9 August 2014 Revised 23 February 2015 Accepted 23 February 2015 